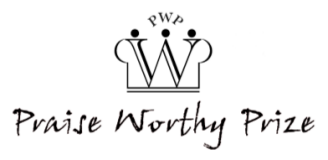

\title{
An Empirical Correlation for the Penetration of a Cryogenic Liquid Jet into a Gaseous Crossflow
}

\author{
Adwaith Ravichandran, Daniel Kirk
}

\begin{abstract}
Empirical correlations have been widely used to map the penetration and trajectory of an injected liquid jet into a gaseous crossflow. Such correlations for the case of cryogenic liquid injection into a gaseous crossflow of the same species are limited. In this study, mediumresolution images of the injection of cryogenic liquid nitrogen into a gaseous nitrogen crossflow with momentum blowing ratios of 1-4 and Weber numbers of 1500-4500 are captured and image analysis is used to identify the jet boundary. Using these data, an empirical correlation for the penetration of liquid nitrogen into a gaseous crossflow as a function of momentum blowing ratio and downstream distance from the point of injection is developed. Minimizing the root mean square error of the correlation parameters over the full range of experimental conditions investigated leads to a power-law correlation with a mean error of $16 \%$. Copyright (C) 2021 The Authors.

Published by Praise Worthy Prize S.r.l. This article is open access published under the CC BY-NC-ND license (http://creativecommons.org/licenses/by-nc-nd/3.0/).
\end{abstract}

Keywords: Injection, Crossflow, Cryogenic, Penetration Depth, Liquid Trajectory

\section{Nomenclature}

$C_{d} \quad$ Liquid orifice discharge coefficient

Do $\quad$ Diameter of liquid orifice [m]

D Test section diameter $[\mathrm{m}]$

$L \quad$ Test section length [m]

$\dot{m}_{g} \quad$ Mass flow rate of gas [kg/s]

$\dot{m}_{l} \quad$ Mass flow rate of liquid [kg/s]

$p_{g} \quad$ Static pressure of gas $[\mathrm{kPa}]$

$\Delta P_{s} \quad$ Driving pressure differential [kPa]

$q$

$T_{g, i}$

$V_{g}$

$V_{l, i n j}$

$W_{l}$

We

$x$

$y$

$\lambda_{s}$

$\rho_{l}$

$\rho g$

$\sigma$

$\mu l$

$\mu g$

RMSE

Momentum blowing ratio $\left(\frac{\rho_{l} V_{l, i n j}^{2}}{\rho_{g} V_{g}^{2}}\right)$

Temperature of gas at inlet [K] injection $[\mathrm{m} / \mathrm{s}]$

Liquid velocity relative to gas $[\mathrm{m} / \mathrm{s}]$

Axial coordinate [m]

Transverse coordinate [m]

Liquid surface wavelength [m]

Liquid density $\left[\mathrm{kg} / \mathrm{m}^{3}\right]$

Gas density $\left[\mathrm{kg} / \mathrm{m}^{3}\right]$

Surface tension $[\mathrm{N} / \mathrm{m}]$

Viscosity of liquid $\left[\mathrm{Ns} / \mathrm{m}^{2}\right]$

Viscosity of gas $\left[\mathrm{Ns} / \mathrm{m}^{2}\right]$

Root Mean Square Error
Gas velocity relative to test section $[\mathrm{m} / \mathrm{s}]$

Liquid velocity relative to test section at

Weber number considering gas phase

\section{Introduction}

The injection of a fluid into a gaseous cross-stream has many engineering applications. For example, the injection of a cool gas to protect turbine surfaces from hot combustion gases is common in high-performance jet engines [1]. In such an application, cold gas is injected on turbine surfaces and blades through a series of holes/slots. A pressure differential between the blade surface and primary flow determines the effectiveness of this cold gas to form a persistent protective film on the blade surface. A sensitivity analysis done as part of the study by Selcuk Uysal [2] concluded that a combination of advanced internal and film cooling technologies is required to reach a gas turbine efficiency of about $65 \%$.

Injecting liquid into gaseous streams has been employed in many applications that include fuel injection in internal combustion engines [3] and the cooling of hot convective gas through direct heat transfer and liquid vaporization. The underlying approach of most liquid injection-based vaporization techniques involve adding mass into a system to decrease the overall mixture temperature. Guerra et al. [4] have employed a Lagrangian approach to track the evolution of an injected liquid spray as it undergoes vaporization and breakup.

The authors have concluded that rapid droplet breakup close to the injection location leads to a predominance of smaller droplets that closely follow the flow downstream.

Since a computationally exhaustive large-eddy simulation is used to model this flow, the need for improved high-resolution modeling at the initial stages of jet injection is stressed by the authors. In many applications, the liquid is injected normally into the mainstream flow to enhance atomization and uniform breakup. Impingement of liquid on the opposing chamber wall or accumulation on injector surface based on the 
liquid kinetic energy at injection is avoided. Studies have also been conducted for liquid injection at differing angles [5] and from varying orifice geometries [6].

Injection of liquid upstream at steep angles results in a longer droplet residence time and helps achieve complete vaporization and energy transfer. The presence of flow swirling devices further enables uniform mixing and complete vaporization. Analysis of differing orifice geometries helps evaluate the effect of orifice discharge coefficient, defined as the ratio between theoretical and actual liquid flow rates across the orifice. In each of these applications, it is vital to determine the trajectory and interface boundary between injected fluid and the ambient fluid. Analytical or relations purely based on first principles are limited in their scope and operating range of validity to calculate such a profile. Therefore, custom correlations based on experimental data have been conventionally used instead. Empirical studies examining crossflow liquid injection into gases have been carried out by Samir Tambe [7]. The correlation between liquid injection velocity, orifice diameter, gas crossflow speed, and transverse penetration depth has been discussed for injection of three different liquids (Water, Jet-A, and N-Heptane). Correlations of jet penetration are also available for experimental investigations of transverse injections in supersonic flows as pursued by Sathiyamoorthy et al. [8] and Anavaradham et al. [9]. The jet trajectory is obtained based on schlieren images which are post-processed further. Such correlations are, however, limited in scope because they are tailored to unique problems of interest.

Moreover, several of these correlations are confined to fluids commonly used in combustion systems (fuels) injected into air/oxidizer flow. Most of the resources mentioned above study injection of liquid from a single orifice. Interaction between tandem liquid jets from one or more orifices injected into a crossflowing fluid has been a recent research area. Sathiyamoorthy et al. [8] have analyzed the effect of tandem-hole configuration on liquid transverse penetration and combustion in supersonic crossflow. They have concluded that higher penetration was achieved for tandem-hole configurations.

Consequently, an increase in spacing between holes/injector orifices increases penetration. The injected liquid column breakup mechanism also determines the type of boundary profile downstream of the injection point. Wu et al. [10] and Andrew et al. [11] have studied this breakup process of liquid jets in subsonic and supersonic crossflows respectively. The former study uses this analysis to obtain curve fit correlations for common liquids in air crossflow. To obtain empirical correlations from an experiment, images captured during this experiment are processed. In most cases, schlieren/shadowgraph images captured using high-speed recording devices track the injection boundary [12]. In this reference, the authors have proposed using 5000 time-resolved experimental images. A standard deviation approach to boundary profile data obtained from these images is implemented. The authors claim that such a method captures low-density droplets in the far-field regions of the spray not visible when profile data from all images are averaged. In the study by Sathiyamoorthy et al. [8], chemiluminescence techniques are used to visualize combustion phenomenon in addition to crossflow injection profile viewed via shadowgraph. This paper examines the injection of a cryogenic liquid into a gaseous stream of the same species under conditions where rapid liquid atomization and significant vaporization occur. Unlike previously mentioned references, the current study is limited to the availability of medium to low-resolution experimental images.

Therefore, these images are processed to a higher extent using a script that employs image recognition and boundary interpretation. This is done by the use of training images and iterative uncertainty minimization.

Section II presents an overview of existing empirical correlations for the trajectory of a liquid injected normal to a gaseous flow. Section III presents an experiment to measure the trajectory of a cryogenic liquid nitrogen jet injected normal to a gaseous nitrogen crossflow over a range of relevant parameters. Section IV presents the image analysis used to determine the shape of the injected liquid trajectory. Section $\mathrm{V}$ develops an empirical correlation for the experimentally collected trajectory data.

\section{Jet Penetration and Trajectory Empirical Correlations}

This section reviews the phenomena that occur when liquid is injected into a gaseous crossflow, including determination of the liquid penetration depth and trajectory. Importance of the momentum-blowing ratio between the two fluids is briefly discussed. The breakup of injected liquid stream into droplets is also reviewed.

Fig. 1 depicts the phenomena when a liquid is injected normal to a gaseous crossflow [12]. The injected liquid exits through a circular orifice of diameter $D_{o}$ and initially behaves as a column. The liquid column thins due to the interaction of viscous, shear, and surface tension forces and then begins to break up into large ligaments and ultimately into smaller droplets. If the injected liquid and the gas are at temperatures where phase change does not occur, and evaporation effects are minimal, the droplets retain their post-break-up size and are carried along with the freestream.

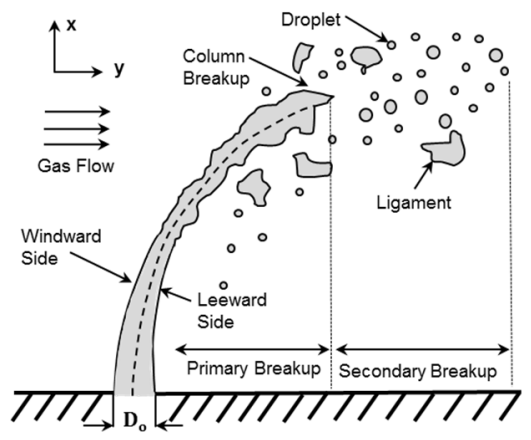

Fig. 1. Breakup process for crossflow liquid injection 
The dashed centerline shows the liquid's overall trajectory and penetration into the gaseous freestream in Fig. 1. The phenomena taking place during the injection, breakup, droplet-formation, and convection process have been well studied, and numerous experimental, analytical and numerical references are available [12], [13], [4].

The momentum-blowing ratio, $q$, is a non-dimensional number that compares the magnitude of momentum between an injected fluid and the mainstream fluid. This non-dimensional number is given in Equation (1):

$$
q=\left(\frac{\rho_{l} V_{l, i n j}^{2}}{\rho_{g} V_{g}^{2}}\right)
$$

In Equation (1), $\rho_{g}$ and $\rho_{l}$ are the gas and liquid phase density while $V_{g}$ and $V_{l, i n j}$ are the gas and liquid velocity.

The momentum blowing ratio is an important parameter that determines the depth of penetration the liquid achieves into the gas crossflow. This wellestablished parameter has been used in many applications [14], [15]. Breakup of liquid column into ligaments, large drops, and ultimately smaller droplets is influenced by dynamic pressure, surface tension, and viscous forces. A second non-dimensional number, the Weber number, is used to characterize the breakup process. Weber number is defined as the ratio of inertial to surface tension forces acting on the liquid. This number quantifies the deforming inertial forces to the reforming surface tension forces that characterize droplet breakup and is given by:

$$
W e=\frac{\rho_{g} W_{l}^{w} D_{o}}{\sigma}
$$

In Equation (2), $\rho_{g}$ is the gas phase density, $D_{o}$ is the liquid orifice diameter, and $\sigma$ is the liquid surface tension. In this study, the liquid velocity relative to cross stream gas velocity $\left(W_{l}\right)$ is used in the Weber number. A Weber number based on the absolute liquid velocity is also used in some literature, predominately for the injection of a liquid into a quiescent medium [16]. The formation of surface waves on the windward side of the liquid jet is responsible for the breakup of the liquid column (Fig. 1). Using the correlation given in [17], the liquid surface wavelength, $\lambda_{s}$, can be estimated as:

$$
\frac{\lambda_{s}}{D_{o}}=3.4 W e^{-0.45}
$$

The correlation given in Equation (3) is valid for $q$ between 3-8000 and $W e>4$. For low $W e(<10)$ injection scenarios, column breakup and bag breakup are dominant, and the liquid surface wavelength can be on the order of the injection orifice diameter or greater. For moderate $W e(\sim 100)$, there are multiple processes responsible for the breakup. The injected liquid stream breakup process for this case can persist over the length of many $D_{o}$ downstream, as shown in Fig. 1. In contrast, for high We $(>1000)$ applications, the injected liquid column is very rapidly broken into small droplets. The current study is concerned with relatively high $\mathrm{We}(>$ 1000), which are similar to those used in sprays and atomizers [18]. For Weber numbers in this study, the liquid surface wavelength to orifice diameter ratio, $\lambda_{s} / D_{o}$, is about $9.5 \%$ and shear force is primarily responsible for liquid column breakup. Weber number and critical velocity have been used to estimate the post-break-up droplet diameter [19]. There are several experimentally based empirical correlations for liquid penetration and trajectory into a gaseous crossflow. The empirical correlations typically take the mathematical form of a power, logarithmic, or exponential curve fit. An example of a power-law correlation is proposed by $\mathrm{Wu}$ et al. [10].

The empirical coefficients are determined using shadowgraph methods, and the resulting correlation is given by:

$$
\frac{y}{D_{o}}=1.37 q^{0.5}\left(\frac{x}{D_{o}}\right)^{0.5}
$$

In Equation (4), $q$ is the momentum blowing ratio, $\mathrm{x}$ and $y$ are axial and transverse coordinates. The range of validity of Equation (4) for $q$ is 3-148, We is 70-1180, $D_{o}$ is $0.5-2.0 \mathrm{~mm}$ when water is injected normally into an air cross-stream. For ethyl alcohol injected into an air cross stream, two momentum ratios of $q=40$ and 98 with $W e=186$ and 461 from a $0.5 \mathrm{~mm}$ orifice were studied. Additional experiments were performed with a mixture of injected liquids. For a mixture composed of 30\% alcohol and $70 \%$ water, the empirical fit is valid over a range of $q=14-147, W e=140-594$, and $D_{o}=0.5-1 \mathrm{~mm}$. For a mixture composed of $40 \%$ glycerol and $60 \%$ water, the empirical fit is valid over a range of $q=15-185$, We $=57$ 287, and $D_{o}=0.5-1 \mathrm{~mm}$. A logarithmic liquid injection profile was proposed by Samir Tambe [7] and is given by:

$$
\frac{y}{D_{o}}=1.55 q^{0.53} \ln \left[1+1.66\left(\frac{x}{D_{o}}\right)\right]
$$

The crossflow air velocity was varied from 76 to 187 $\mathrm{m} / \mathrm{s}$ with liquid Jet-A normally injected at a velocity of 7 to $16 \mathrm{~m} / \mathrm{s}$, resulting in a momentum ratio range of 5-10.

The Weber number ranges from 95-1150 for these cases. An example of a correlation involving exponential terms was proposed by Chen et al. [20] and is given by:

$$
\begin{gathered}
\frac{y}{D_{o}}=9.91 q^{0.44}\left[1-e^{-\left(x / D_{o}\right) / 13.1}\right] \cdot \\
\cdot\left[1+1.67 e^{-\left(x / D_{o}\right) / 4.77}\right]\left[1+1.06 e^{-\left(x / D_{o}\right) / 0.86}\right]
\end{gathered}
$$

The correlation is valid for fuel injection into crossflowing air with $q$ between $8-45, D_{o}$ between 0.25 $1.77 \mathrm{~mm}$. The correlations in Equations (4), (5), and (6) consider momentum ratio as the only parameter that sets the shape of injection trajectory. However, other published correlations include additional density and viscosity effects of fluids. For crossflow injections 
dominated by viscous and shear breakup effects, Stenzler et al. [21] proposed the following correlation, which includes the influence of viscosity ratio of the fluids:

$$
\frac{y}{D_{o}}=2.630 q^{0.44}\left(\frac{x}{D_{o}}\right)^{0.39}(W e)^{-0.088}\left(\frac{\mu_{l}}{\mu_{g}}\right)^{-0.027}
$$

In Equation (7), $\mu_{l}$ and $\mu_{g}$ are the viscosity of the liquid and gas. This expression is applicable for the injection of water into air crossflow with $q$ between 18$36, W e$ between $2-40$, and $D_{o}=0.254 \mathrm{~mm}$. Fig. 2 shows a plot of the Equations (4), (5), and (6). The plot shows the non-dimensional penetration depth $\left(y / D_{o}\right)$ as a function of non-dimensional distance $\left(x / D_{o}\right)$ for momentum ratios of $q=10$ and 50 .

Fig. 2 shows that closer to the point of injection for $x / D_{o}<20$, the penetration depth for the logarithmic and exponential profiles increases more rapidly with downstream distance than the power-law profile prediction. Further away from injection for $x / D_{o}>20$ the penetration predicted by power-law increases more rapidly with downstream distance. At $q=10$ and $x / D_{o}=100$, the power-law predicts a penetration of $y / D_{o}=43$, while the logarithmic and exponential profiles predict a penetration of $y / D_{o}=26$ and 27, respectively. As the momentum ratio is increased to $q=50$ at the same value of $x / D_{o}=100$, the power-law predicts penetration of $y / D_{o}=97$, while the logarithmic and exponential laws predict the penetration of 63 and 55, respectively. The exponential profile predicts a maximum value of penetration, and then the penetration distance decreases further downstream. For example, the maximum penetration depth for $q=10$ is $y / D_{o}=27$ at $x / D_{o}=136$, and for $q=50$, the maximum penetration depth is $y / D_{o}=55$ at $x / D_{o}=141$. The correlations available in the literature are typically for values of $q$ that are in the range of 10 to 100 , and minimal data exists for liquid into gas stream injection at lower momentum ratios. Further, the injection of cryogenic nitrogen into a warm gaseous nitrogen stream also results in phase change, in which the liquid droplets vaporize, adding an additional process to the phenomena shown in Fig. 1.

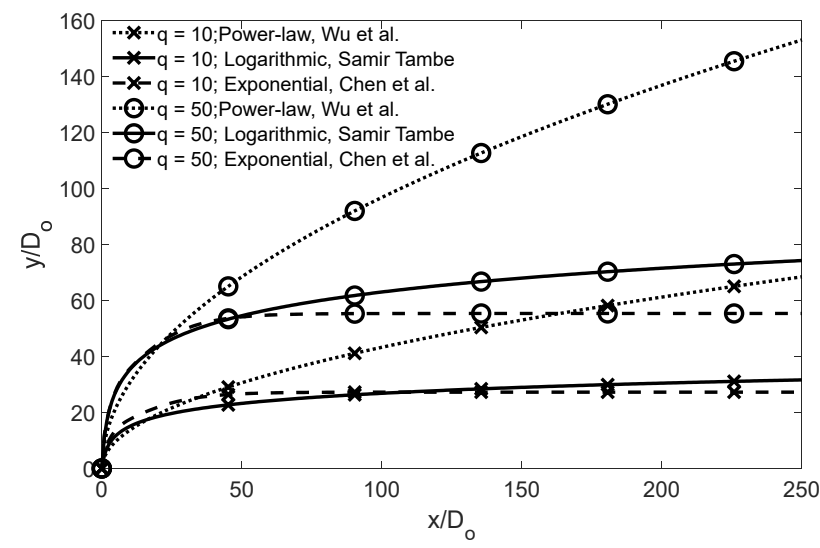

Fig. 2. Power law, logarithmic law, and exponential injection trajectory correlations for $q=10$ and 50
As the liquid changes phase, the boundary between the injected liquid and gaseous stream becomes less discernable, and both the momentum and thermal boundaries between the jet and freestream may be relevant. For these reasons, it was desirable to conduct experiments to measure cryogenic liquid penetration into a warm gaseous cross-stream over a range of lower momentum ratios and ascertain if the existing literature curve fits can be adequately used to characterize the trajectory of the liquid jet.

\section{Experimental Overview}

An experiment was performed to measure the penetration of a liquid nitrogen jet that is injected normal to a gaseous nitrogen crossflow over a range of momentum ratios of interest. A schematic of the experiment and an image of liquid nitrogen injection into a gaseous nitrogen crossflow are shown in Figs. 3. In the experiment, nitrogen gas flows at the desired velocity and pressure. Liquid nitrogen is injected normal to the flow through 100 injection orifices each with diameter $D_{o}$ and penetrates the gaseous nitrogen crossflow. The orifices are in a helical pattern on the center body. The pitch of this helix is $3.2 \mathrm{~mm}$ with seven revolutions spanned by all 100 orifices. Each revolution approximately contains 14 orifices which are uniformly staggered circumferentially. A transparent acrylic test section allows for the capture of high-speed images. Image analysis is used to determine the shape of the injected liquid trajectory. The test section is cylindrical with diameter, $D$, of $76.2 \mathrm{~mm}$. The orifice diameter, $D_{o}$, is $0.79 \mathrm{~mm}$. The overall length of the test section downstream of the point of injection, $L$, is $700 \mathrm{~mm}$, which corresponds to an $x / D_{o}$ of 900 and $L / D$ of 9 . The center body diameter is $25 \mathrm{~mm}$ and extends $80 \mathrm{~mm}$ past the last row of orifices. The center body is tapered after the last row of orifices to prevent the formation of recirculation zones.

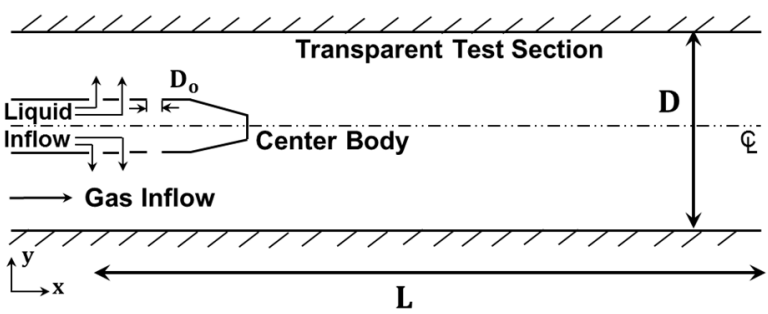

(a)

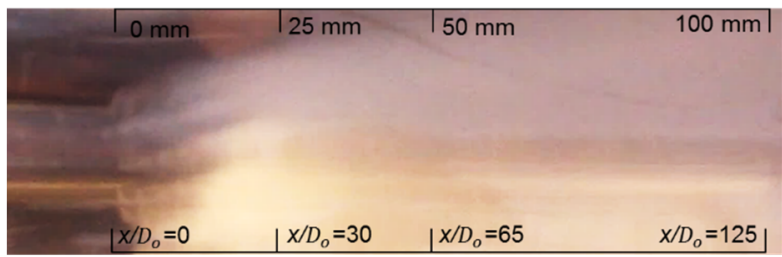

(b)

Figs. 3. Test section schematic (a) and image showing injection of liquid nitrogen into gaseous nitrogen crossflow at $q=2.8$ (b) 
This experiment was designed to study an application in which cylindrical symmetry and circumferential uniformity of the injection process was desired, as compared with single, discrete orifice injection which are more common in the literature. The experimental conditions are summarized in Table I. The ratio of the liquid to gas flow rate is given by $\dot{m}_{l} / \dot{m}_{g}$. To determine the momentum ratio, $q$, the velocity of the gas and liquid must be known. The velocity of the gas at the point of injection is calculated through Equation (8):

$$
V_{g}=\frac{\dot{m}_{g}}{\rho_{g} A}
$$

In Equation (8), $\dot{m}_{g}$ is the measured gas flow rate. The cross-sectional area of the test chamber $A$ is known. The gas density $\rho_{g}$ is calculated using curve fit data available for various cryogenic thermo-fluids, based on the test section static pressure and temperature which are measured. Nitrogen gas temperature and pressure are measured using a thermocouple and pressure transducer, respectively, located in the test section. The velocity of liquid at injection point is determined from Equation (9):

$$
V_{l, i n j}=\frac{\dot{m}_{l}}{\rho_{l} A_{o} N C_{d}}
$$

In Equation (9), $\dot{m}_{l}$ is the measured liquid mass flow rate, $N$ is the number of injector orifices, $A_{o}$ is the injection orifice area, $\rho_{l}$ is the density of the liquid, and $C_{d}$ is the discharge coefficient associated with the liquid injection orifice.

The temperature of the injected liquid nitrogen is $80 \mathrm{~K}$ and the liquid density is $792.6 \mathrm{~kg} / \mathrm{m}^{3}$.

Liquid nitrogen temperature is measured using a thermocouple located in the source tank. Injection orifices are fabricated using electrical discharge machining and do not have a sharp edge. Using measured liquid flow rate, discharge coefficient for orifices is experimentally determined. The average discharge coefficient for all 3 cases is 0.21 with a standard deviation of 0.06 . The measured liquid flow rate is related to orifice area and driving pressure difference, as given by Equation (10):

$$
\dot{m}_{l}=A_{o} N C_{d} \sqrt{2 \rho_{l}\left(p_{l}-p_{g}\right)}
$$

In Equation (10), $\Delta P_{s}\left(p_{l}-p_{g}\right)$ is the static pressure difference between the liquid in the center body and the nitrogen gas above at the injection location.

TABLE I

EXPERIMENT TEST CONDITIONS

\begin{tabular}{cccccc}
\hline \hline Case & $\frac{\dot{m}_{l}}{\dot{m}_{g}}$ & $q$ & $T_{g, i} T_{g, i}(\mathrm{~K})$ & $W e$ & $\left(\frac{x}{D_{o}}\right)_{\text {vap }}$ \\
\hline 1 & 0.08 & 1.3 & 240 & 4036 & 287 \\
2 & 0.20 & 2.8 & 234 & 4428 & 765 \\
3 & 0.27 & 4.1 & 189 & 1521 & 1504 \\
\hline \hline
\end{tabular}

Using the velocity and density, the injection momentum ratio can be determined, and are also summarized in Table I. Based on the cryogenic thermofluid model proposed by Ravichandran et al. [22], the distance required for complete evaporation for injected liquid is at an $x / D_{o}$ of 287 for case 1 and $x / D_{o}=765$ for case 2. Case 3 results in incomplete evaporation within the length of the test section, but full vaporization would occur at $x / D_{o}>1500$.

These lengths are also summarized in Table I. Because the momentum ratio and Weber number are not measured directly, the individual measurement errors of each quantity must be compounded when determining experimental uncertainty in these parameters. The mass flow rates of gaseous and liquid flows are measured directly using a mass flow meter with an error of $0.5 \%$.

The error in temperature measured using platinum resistance temperature detectors is $0.25^{\circ} \mathrm{C}$ or $0.5 \%$ [23].

The uncertainty in pressure measurement using a pitot-static probe is $0.5 \%$ [24], [25]. The error in crosssectional area of test section is $0.5 \%$, and the error in the size of the injection orifice is $2 \%$. The error in $V_{g}$ and $V_{l, i n j}$ is $1.2 \%$ and $2.3 \%$, respectively. The overall uncertainty in $q$ is $5.4 \%$ and the overall uncertainty in $W e$ is $3.5 \%$.

\section{Results}

A high-speed camera is used to capture images of the liquid injection, breakup, and droplet vaporization processes. Figs. 3 showed an image from the experiment for case 2. The injection of liquid nitrogen presents a visual contrast against the gaseous crossflow. Although the images show a discernable liquid injection trajectory, the exact location of the boundary is subjective. Image analysis, along with a specified jet boundary gradation criterion, provides a method to quantitatively determine the injection boundary. Each image is preprocessed to make identification of the injected jet boundary more apparent.

The location of the orifices and test section are demarcated in the images. Colored images are converted to monochrome by applying a grayscale field filter to the red, blue and green plane composites of the original image. Using the monochrome images, the Domain of Interest (DOI) is demarcated. Pixel glare and shadow are next removed within the DOI. To determine the exact greyscale value at which liquid-gas phase domain transition occurs, a computer vision tool is used to train the script to find pixels that represent liquid with greater than $90 \%$ confidence. This process is performed on highresolution training images, and the procedure is repeated iteratively for several trials. Using this approach, the jet boundary is quantitatively defined. Examples of similar approaches to defining the boundary between two fluid jets can be found in [26] and [27]. Fig. 4 shows postprocessed images for the test cases. Using image analysis, several injection profiles from different orifices can be simultaneously tracked. 


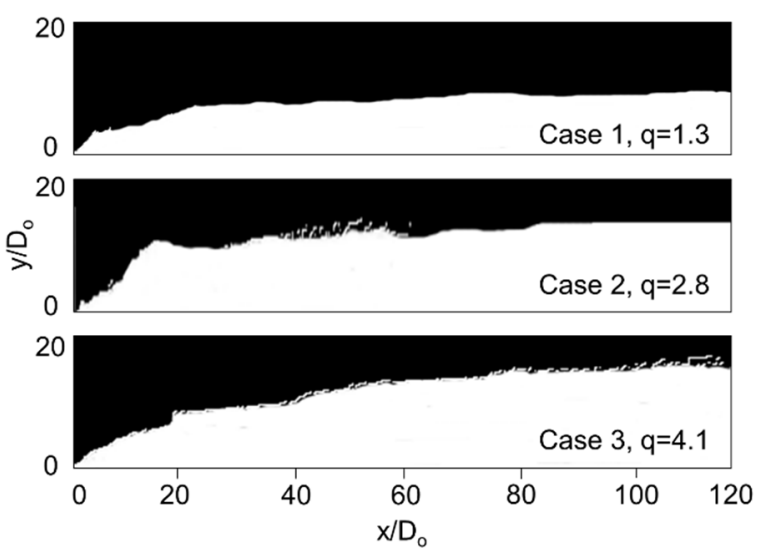

Fig. 4. Processed image to obtain injection boundary for case 1 (top), case 2 (middle), and case 3 (bottom)

Key factors influencing accurate boundary determination include flash vaporization effects and ambient specular reflection on the translucent test section wall. Fig. 5 shows the experimentally extracted trajectory data from the image analysis plotted non-dimensionally as $x / D_{o}$ vs. $y / D_{o}$ for the cases shown in Table I. The results from Fig. 5 demonstrate that the multi-orifice injection boundary profile could be divided into two zones: the near-field zone axially encompasses the 100 orifices along the center body (spanning $x / D_{o}$ between $0-60)$, and the far-field zone where the interaction between injections from different orifices has effectively merged and the injection trajectory behaves as if it emanated from a single orifice (spanning $x / D_{o}>60$ ).

Cases 2 and 3 in Fig. 5 illustrate this behavior, whereas for the lower blowing ratio of case 1 , the individual jets coalesce closer to the location of the first few orifices. Although the correlations presented in Equations (4)-(7), were not generated over a similar range of momentum ratios to those considered in the present study, the correlations were based on comparable Weber numbers in which shear is responsible for liquid column breakup [17]. Figs. 6 compare the experimental data with the power law (Equation (4)), logarithmic form (Equation (5)), exponential form (Equation (6)), and Weber number based (Equation (7)) correlations.

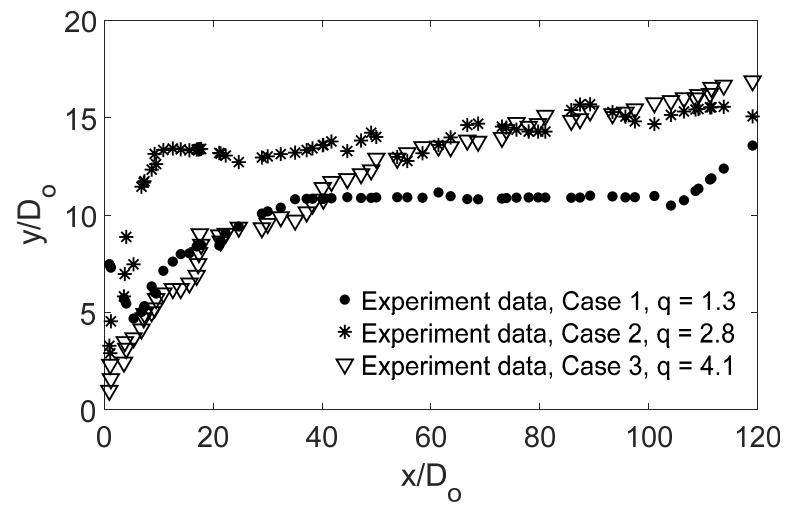

Fig. 5. Experiment data using image processing

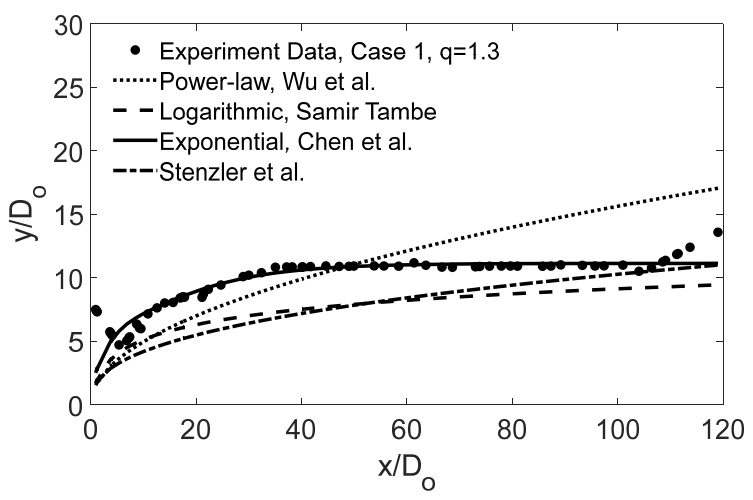

(a)

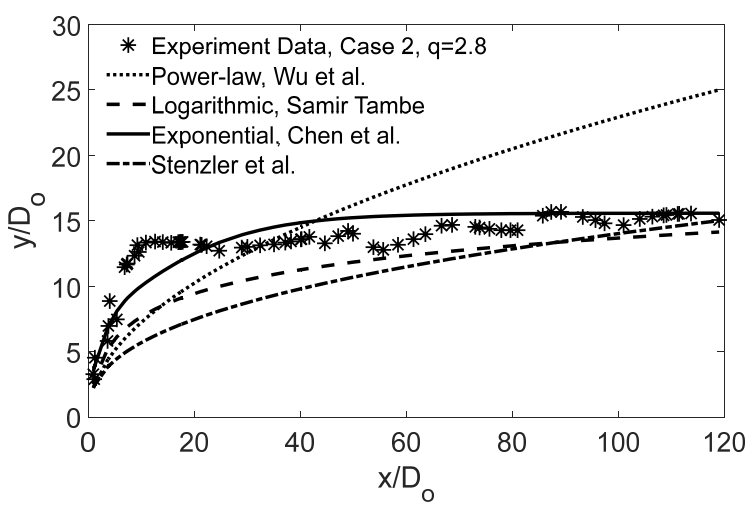

(b)

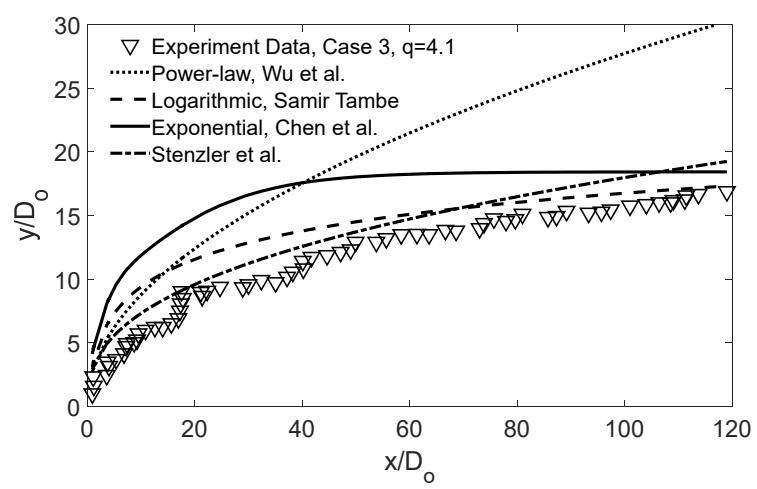

(c)

Figs. 6. Comparison of existing profiles to experiment data for case 1 (a), case 2 (b), and case 3 (c)

Table II presents the error between the experimental data and the existing injection profile correlations. The weak agreement between the collected data and the existing correlations is not unexpected as the range of blowing ratios, multi-orifice injection geometry, and cylindrical test section are different from experimental conditions in which the correlations were derived.

TABLE II

Percentage Error Between Literature Predictions And EXPERIMENTALLY MEASURED DATA

Error with Experiment Data, \%

Case $q$ Power law Eq. Log-law Eq. Exp Form Weber Eq.

\begin{tabular}{cccccc} 
& & $(4)$ & $(5)$ & Eq. (6) & $(7)$ \\
\hline 1 & 1.3 & 39 & 45 & 12 & 52 \\
2 & 2.8 & 35 & 27 & 10 & 48 \\
3 & 4.1 & 38 & 22 & 35 & 16 \\
\hline \hline
\end{tabular}


In order to increase the predictive capability of the power-law and logarithmic form, new coefficients are determined for these correlations in the next section.

These two types of correlations are explicitly chosen to minimize the number of regression constants to be solved and arrive at compact trajectory equations.

\section{Proposed Empirical Correlation for Cryogenic Liquid Nitrogen Injection}

This section uses the jet trajectory profiles derived from the image analysis to determine the coefficients in the power-law and logarithmic form correlations that best fit the conditions summarized in Table I. The form of power-law is given by Equation (11), where the coefficients $A$ and $n$ are to be determined experimentally:

$$
\frac{y}{D_{o}}=A q^{n}\left(\frac{x}{D_{o}}\right)^{n}
$$

Similarly, the coefficients $B, m$, and $C$ are determined for the logarithmic form, which is given by Equation (12):

$$
\frac{y}{D_{o}}=B q^{m} \ln \left[1+C\left(\frac{x}{D_{o}}\right)\right]
$$

The empirical coefficients are obtained using regression analysis where the individual Root Means Square Error (RMSE) is minimized. This RMSE is calculated between $y / D_{o}$ of experimental data and that based on Equations (11) and (12) by varying the coefficient values. The root mean square error is given by:

$$
R M S E=\sqrt{\sum_{j=1}^{h}\left[\frac{1}{g} \sum_{i=1}^{g}\left(\left(\frac{y}{D_{o}}\right)_{i, \text { exp }}-\left(\frac{y}{D_{o}}\right)_{i, \text { eqn }}\right)^{2}\right]}
$$

where $g$ corresponds to the number of data points in a case, and $h$ corresponds to the number of cases (data sets) used in the error minimization. For the individual case minimization, $h=1$. The values that minimize the error for each case are summarized in Table III. Table III also presents the error, $E r$, between the experimental data and the prediction made by the best fit coefficients for the power-law and logarithmic form. The power law and logarithmic form all fit the data to better than $10 \%$ error for each case. Figs. 7 show the three cases with best-fit curves using simultaneous minimization of Equation (13) as objective function with $h=3$ for power and logarithmic form.

TABLE III

EXPERIMENTALLY DETERMINED EMPIRICAL CURVE FIT PARAMETERS

\begin{tabular}{ccccccccc}
\hline \hline \multirow{2}{*}{ Case } & \multicolumn{4}{c}{ Power Law } & \multicolumn{4}{c}{ Logarithmic Law } \\
& & A & $n$ & $E r \%$ & $B$ & m & $C$ & $E r \%$ \\
\hline 1 & 1.3 & 4.7 & 0.19 & 9 & 1.4 & 0.42 & 14.0 & 9 \\
2 & 2.8 & 6.4 & 0.16 & 9 & 1.4 & 0.35 & 18.5 & 8 \\
3 & 4.1 & 1.2 & 0.43 & 6 & 1.7 & 0.81 & 0.19 & 6 \\
\hline \hline
\end{tabular}

The power-law coefficients are $A=3.86 ; n=0.24$, and log-law coefficients are $B=2.26 ; m=0.162 ; C=2.14$. As noted earlier, due to the multi-orifice injection geometry, two zones of jet behavior can be observed.

The curve fit values in the near field to the injection zone, spanning an $x / D_{o}$ between $0-60$, have a greater deviation from the power-law and logarithmic curve fit forms, which are based on single, discrete injection jet studies. In the current study, the multiple jets interact and do not behave as a single discrete jet in this near field injection zone. Curve fit values further away from this zone better predict the experimental data, as the penetration profile begins to exhibit far-field behavior from a single jet.

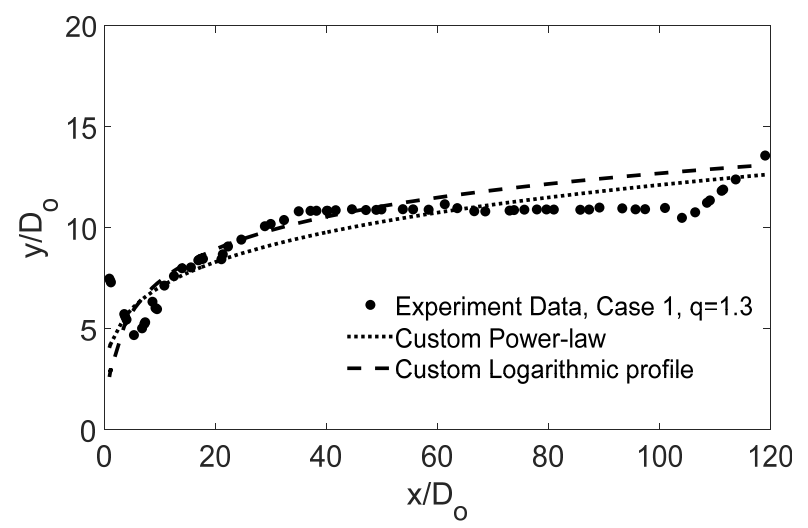

(a)

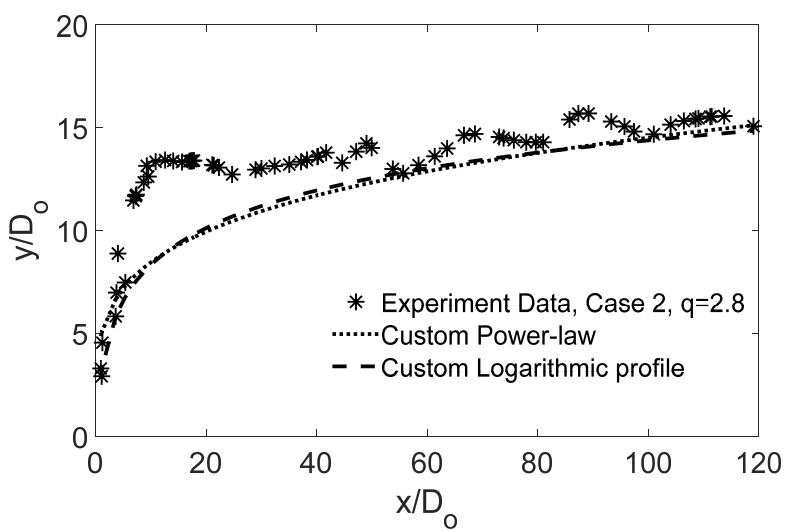

(b)

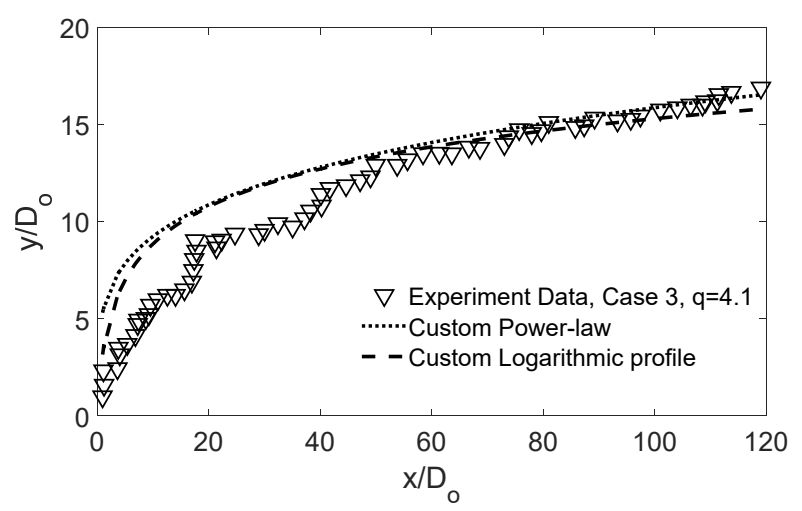

(c)

Figs. 7. Custom curve fit correlations for case 1 (a), case 2 (b), case 3 (c) 
For case 1, both the power-law and logarithmic form correlations track the overall shape of jet trajectory into the freestream. In case 2, the correlations track the trajectory of the jet near the vicinity of the injection orifice $\left(0<x / D_{o}<5\right)$ and then underpredict the penetration of the jet between $5<x / D_{o}<60$ with a mean error of $28 \%$ for both laws in this zone. Beyond $x / D_{o}>$ 60 , the correlation again tracks the shape of the penetration with a mean error of $5 \%$ for both correlations. For case 3, the correlation over predicts the jet penetration into the freestream for $x / D_{o}<50$ with a mean error of $29 \%$ and $26 \%$ for the power and logarithmic form, respectively, and then tracks the shape of the profile for $x / D_{o}>50$ with errors of $2.2 \%$ and $2.5 \%$.

The overall mean error is $10 \%$ for the custom power law and $14 \%$ for the logarithmic form using the custom fit coefficients with experiment data in both zones for case 1 . For case 2, the corresponding errors in the near field and far field are $19 \%$ and $19 \%$, respectively. For case 3 , the errors in the near field and far field are $19 \%$ and $17 \%$, respectively. The overall average error for all three cases using both correlations is $16 \%$. The compounding of experimental measurement errors leads to an uncertainty of $\pm 5.4 \%$ on $q$. The correlations can be used to examine the sensitivity of injection trajectory with uncertainty in $q$. For example, if $q$ is only known to within $\pm 30 \%$, the injection profile boundary can be determined using Equation (11). Fig. 8 shows the powerlaw applied to case 2, assuming $q=3.64 \quad(+30 \%$ uncertainty on $q$ ) and $q=1.96$ (-30\% uncertainty on $q)$.

For $q=2.8$, the correlation predicts a value of $y / D_{o}=8.6$ at $x / D_{o}=10$, however, with $30 \%$ uncertainty on $q$, the predicted $y / D_{o}$ can range from 7.9 to 9.2 . At $x / D_{o}=60$, the range of uncertainty on $y / D_{o}$ is $11.9-13.8$, while the correlation predicts a penetration of $y / D_{o}=12.9$. At $x / D_{o}=100$, the range of uncertainty on $y / D_{o}$ is $13.4-15.5$, while the correlation predicts a penetration of $y / D_{o}=14.5$.

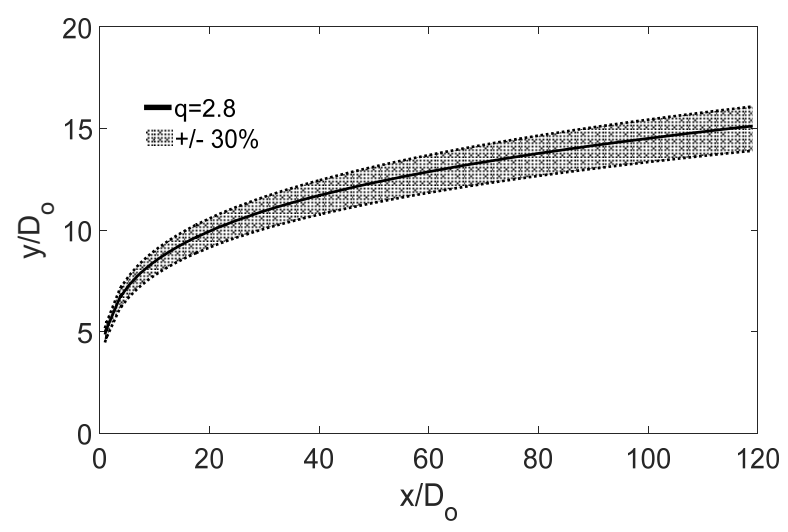

Fig. 8. Jet penetration as a function of downstream distance for $\pm 30 \%$ experimental uncertainty on momentum ratio of $q=2.8$

\section{Conclusion}

The injection of liquids into a gaseous crossflow has many applications in aerospace engineering. However, there is limited data available regarding the injection of cryogenic liquids into a gaseous crossflow, especially for moderate momentum blowing ratios of less than five and high Weber numbers in which the breakup of the injected jet occurs via shear. In this study, an experiment was conducted over a range of momentum blowing ratios of 1.3-4.1 and Weber numbers greater than 1,000. Image analysis was used to determine the jet penetration trajectory as a function of downstream distance from the point of injection for three different momentum blowing ratios. The empirical coefficients for both a power law and logarithmic form were determined, and the correlations developed as a result of this work have a mean error of $16 \%$ for momentum blowing ratios between 1.3 and 4.1. Future work will focus on extending the experimental efforts to other cryogenic liquid/gas combinations, including hydrogen and oxygen. Subsequent experimental work will also consider dissimilar liquid/gas combinations with liquid nitrogen injection into gaseous helium and argon. In these studies, the momentum blowing ratio will be kept constant for different types of gases in order to determine if there is a strong dependence on the penetration trajectory due to the viscosity ratio between the injected liquid and crossflow gas. The authors intend to expand upon the experimental range of momentum blowing ratios from 0.5 to 10 and Weber numbers from 100 to 10,000 to extend the range of applicability of the power-law correlation. Finally, the experiment will also be upgraded with an additional camera to collect images from above in order to determine a correlation of the lateral spread of the injected liquid jet. Information about the lateral spreading is important for multi-hole injection strategies used for cooling applications.

\section{References}

[1] L. Cheng, H. Zhu, R. Jiang, Z. Fu and W. Xu, The Effect of Turbulence Intensity on Full Coverage Film Cooling for a Turbine Guide Vane, in AIAA Propulsion and Energy Forum, Cincinnati, 2018

[2] S. C. Uysal, Analysis of Gas Turbine Cooling Technologies for Higher Natural Gas Combined Cycle Efficiency, in AIAA Propulsion and Energy Forum, Virtual Event, 2020.

[3] A. P. Singh, N. Sharma, V. Kumar, D. P. Satsangi and A. K. Agarwal, Fuel Injection Strategy for Utilization of Mineral Diesel Methanol Blend in a Common Rail Direct Injection Engine, Journal of Energy Resources Technology, vol. 142, p. 13, 2020.

[4] J. T. Guerra, J. R. Edwards and K.-C. Lin, Droplet Vaporization and Secondary Breakup Effects in Aerated-Liquid Injection into a Supersonic Crossflow, in AIAA SciTech Forum, Virtual Event, 2021.

[5] V. G. Awate and P. J. Ansell, Characterization of Inclined Oscillating Jet and Crossflow Interaction for Use in Active Flow Control, in AIAA SciTech Forum, Orlando, 2020.

[6] X. Wei, G. Zhengyan and C. Pimin, Effect of Geometric Parameters on Mean Drop Sizes From Dual-Orifice Pressure Nozzles, Journal of Engineering for Gas Turbines and Power, vol. 141, p. 6, 2019.

[7] S. Tambe, Liquid Jet in Subsonic Crossflow, Division of Research and Advanced studies- The University of Cincinnati, Cincinnati, 2004.

[8] K. Sathiyamoorthy, T. H. Danish, V. S. Iyengar, J. Srinivas, X. Harikrishna, T. M. Muruganandam and S. R. Chakravarthy, Penetration and Combustion Studies of Tandem Liquid Jets in Supersonic Crossflow, Journal of Propulsion and Power, vol. 36, 
no. 6, p. 11,2020

[9] T. Anavaradham, O. Shreenivasan, R.I.Sujith, S. Chakravarthy and S. Panneerselvam, An Experimental Investigation of Transverse Liquid Injection in Confined Supersonic Flow over Cavities, in AIAA/CEAS Aeroacoustics Conference, 2004.

[10] P.-K. Wu, K. A. Kirkendall and R. P. Fuller, Breakup processes of liquid jets in subsonic crossflows, Journal of Propulsion and Power, p. 13, 1997.

[11] A. M. Hess, D. A. Kessler, R. F. Johnson, G. B. Goodwin, C. Aguilera and J. Sosa, Evaluation of Droplet Aerodynamic Breakup Models in Supersonic and Hypersonic Flows, in AIAA SciTech Forum, Virtual Event, 2021.

[12] V. G. Shaw, R. Holpp, N. Stocker, B. Wonziak, J. Coffey, A. Gaetano, T. Pritschau and E. J. Gutmark, Breakup Characteristics and Far-Field Trajectory of Liquid Jets in Subsonic Crossflow, in AIAA Propulsion and Energy Forum, Virtual Event, 2020.

[13] S. Salauddin, W. Flores, M. Otero and K. Ahmed, Modal Analysis of Liquid Fuel Jet in Crossflow, in AIAA Propulsion and Energy Forum, Virtual Event, 2020.

[14] F. Ladeinde, Effect of Momentum Flux Ratio on Combustion in the Hyshot Scramjet Engine, in AIAA Propulsion and Energy Forum, Indianapolis, 2019.

[15] Y. D. Won, Y. H. Cho, S. W. Lee and W. S. Yoon, Effect of Momentum Ratio on the Mixing Performance of Unlike Split Triplet Injectors, Journal of Propulsion and Power, vol. 18, no. 4, p. $8,2002$.

[16] Z. S, B. Milton, K. Pianthong and B. M, Supersonic liquid fuel jets injected into quiescent air, Internation Journal of Heat and Fluid Flow, 2004.

[17] K. A. Sallam, C. Aalburg and G. M. Faeth, Breakup of Round Nonturbulent Liquid Jets in Gaseous Crossflow, AIAA Journal, vol. 42, no. 12, p. 12, 2004.

[18] Y. S. Hwang and Y. i. Jin, The penetration characteristics of the normally injected kerosene liquid jet in high Weber number flow, Joint Propulsion Conference \& Exhibit, vol. 45, p. 6, 2009.

[19] A. H. Lefebvre, Atomization and Sprays, West Lafayette: Taylor and Francis, 1989.

[20] T. H. Chen, C. R. Smith and D. G. Schommer, Multi-Zone Behaviour of Transverse Liquid Jet in High Speed Flow, in Aerospace Sciences Meeting \& Exhibit, Reno, 1993.

[21] J. N. Stenzler, J. G. Lee and D. A. Santavicca, Penetration of Liquid Jets in a Crossflow, in Aerospace Sciences Meeting and Exhibit, Reno, 2003.

[22] Ravichandran, A., Storey, J., Kirk, D., A Thermo-Fluid Model of Droplet Evaporation and Pressure Variation in Venturi LiquidGas Mixers, (2020) International Review of Aerospace Engineering (IREASE), 13 (3), pp. 108-119. doi: https://doi.org/10.15866/irease.v13i3.18758

[23] T. Oishi, S. Gorbunov, J. Fu and E. Martinez, Thermal Sensor Development and Testing for NASA Entry Probe Thermal Protection Systems, in AIAA Aerospace Sciences Meeting and Exhibit, Reno, 2006.

[24] Surrey Sensors, 17 October 2017. [Online, Accessed 1 January 2021].

Available: https://www.surreysensors.com/article/uncertain-pitotstatic-probe/

[25] United Sensor, [Online, Accessed 1 January 2021]. Available: https://www.unitedsensorcorp.com/pitot-properties.html

[26] R. Zakaria, P. Bryanston-Cross and B. Timmerman, Digital image processing techniques for the analysis of fuel sprays global pattern, in IOP Conference Series: Materials Science and Engineering, 2017.

[27] S. Eppel and T. Kachman, Computer vision-based recognition of liquid surfaces and phase boundaries in transparent vessels, with emphasis on chemistry applications, Haifa.

\section{Authors' information}

Department of Aerospace, Physics and Space Sciences, Florida Institute of Technology.

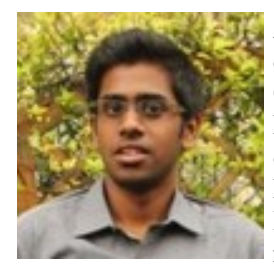

Adwaith Ravichandran completed his B.E. degree in aeronautical from Kumaraguru College of Technology, India in 2015 and an M.S. degree in aerospace from Florida Institute of Technology in 2018. Currently pursuing a $\mathrm{Ph.D.}$ in aerospace engineering from Florida Institute of technology, he has been working on numerical and analytical modelling of multiphase systems. He has presented a paper at an international conference held in Paris, France on gas turbine diffusers as part of his undergraduate studies and is also a student member of the AIAA.

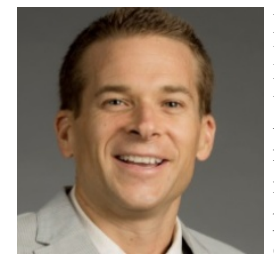

Dr. Daniel R. Kirk is a Professor of Aerospace Engineering at Florida Institute of Technology. Dr. Kirk received his Ph.D. from the MIT in Aeronautics and Astronautics. Dr. Kirk's research focuses on rocket propulsion and modeling of thermal behavior of liquid rocket propellants. Dr. Kirk is associate fellow member of the AIAA. 